\title{
SIMULATION OF NANO-SCALE CUTTING WITH MOLECULAR DYNAMICS
}

\author{
Angelos P. Markopoulos, Kalliopi-Artemi L. Kalteremidou and Dimitrios E. Manolakos \\ Department of Manufacturing Technology, School of Mechanical Engineering, \\ National Technical University of Athens, Heroon Polytechniou 9, 15780, Athens, Greece
}

Received 2014-11-22; Revised 2014-11-24; Accepted 2015-04-20

\begin{abstract}
The simulation of nanometric cutting of copper with diamond cutting tools, with the Molecular Dynamics method is considered. A 2D model of orthogonal nano-scale cutting is presented and the influence of the depth of cut and tool rake angle on chip morphology and cutting forces is investigated. For the analysis, three different depths of cut, namely $10 \AA, 15 \AA$ and $20 \AA$ and four tool rake angles, namely $0^{\circ}, 10^{\circ}, 20^{\circ}$ and $30^{\circ}$ are tested. Results indicate that with increasing depth of cut, cutting forces also increase, while with increasing tool rake angle, cutting forces decrease. Furthermore, the effect of Lennard-Jones and Morse potentials on final results of the simulation is studied and discussed. The proposed model can be successfully used for the modeling and simulation of cutting operations that continuum mechanics cannot be applied or experimental and measurement techniques are subjected to limitations or it is difficult to be carried out.
\end{abstract}

Keywords: Simulation, Molecular Dynamics, Nano-Scale Cutting, Lennard-Jones and Morse Potentials

\section{INTRODUCTION}

In recent years, the development of nanotechnology has been extremely high. With nanotechnology, a big variety of miniaturized mechanical parts can be produced. These mechanical parts can be used in a wide range of applications, including computer systems, communication systems, aerospace, medical equipment.

In order to manufacture parts with dimensions of nanometres, high-technology equipment and cutting tools are required. These manufacturing processes cost a lot and aim to extremely small dimensions. The ultra-precision machining with diamond tools can be used in order to produce sub-micrometer form accuracy and a few nanometers surface roughness at acceptable cost levels.

There is a big need to study the nanometric cutting mechanisms and all the new phenomena that may appear in this scale. This is extremely difficult via experiments, as they cost a lot and may not be sufficiently efficient for the aimed dimensions. Another way is to study the aforementioned manufacturing processes and phenomena using computational methods. Nanoscale cutting involves workpiece deformation in only a few atomic layers at the surface. On this scale, the continuum theory cannot be used, so methods like finite elements cannot be used, too. On this scale, a method that can be used for study on the atomic level is Molecular Dynamics.

In Molecular Dynamics, the workpiece and the cutting tool are analysed in atoms, taking into consideration the structure of each material and applying specific boundary conditions. The method of Molecular Dynamics uses specific interatomic potentials and Newtonial dynamics. The Newtonian equations of motion are applied to each atom of the system and then they are solved using numerical methods. The forces that are developed on the atoms are calculated from the interatomic potential used. In order to compute the force acting on a specific atom of the system, all the forces contributed by the surrounding atoms are summed (Rapaport, 2004).

Several researchers use Molecular Dynamics simulation to study nanometric cutting; Komanduri and Raff (2001) offer a thorough review. Komanduri et al. Corresponding Author:Angelos P. Markopoulos, Department of Manufacturing Technology, School of Mechanical Engineering, National Technical University of Athens, Heroon Polytechniou 9, 15780, Athens, Greece 
(1998; 1999; 2000; 2001) studied nanometric cutting of single-crystal aluminum and tried to understand the effects of crystal orientation and tool geometry on the cutting mechanisms. Ikawa et al. (1991) and Shimada (1995) investigated the effect of tool edge radius and depth of cut on the chip formation mechanism using a two-dimensional system. Kim and Moon (1996) used the Morse potential in order to simulate the cutting mechanism of copper and aluminium using a diamond tool. Ye et al. (2003) used the EAM potential in order to study the single-crystal copper and compute the interatomic forces. Pei et al. (2006) compared the use of Morse potential and EAM potential on Molecular Dynamics simulation of cutting copper using different tool rake angles. A large number of the existing studies use two-dimensional systems and study the developed energy and cutting forces, as well as the chip formation mechanism and the various phenomena.

In the present study, based on finding and data of previous works, a nano-scale model of cutting of copper with diamond tools is presented. The models are realized in Matlab ${ }^{\circledR}$ and several simulations are performed in order to investigate the influence of depth of cut and tool rake angle on the cutting forces and chip geometry. Furthermore, the use of different potentials pertaining to the interactions between the atoms of the analysis is investigated and useful conclusions are drawn.

\section{MATERIALS AND METHODS}

The 2D simulation model presented consists of a copper workpiece and a diamond tool as shown in Fig. 1. It is a relatively small system, with the workpiece consisting of 800 atoms, following the fcc structure of copper and using the lattice constant of copper, which is $3.62 \AA$. The tool consists of 120 atoms and the edge of the tool is straight and perpendicular to the direction of motion. Tool clearance angle $\beta$ is kept at $0^{\circ}$. In this study, four different tool geometries with the rake angle $\alpha$ being $0^{\circ}, 10^{\circ}, 20^{\circ}$ and $30^{\circ}$, respectively, are used. The cutting speed is $123 \mathrm{~m} / \mathrm{sec}$ and the depth of cut is $10 \mathrm{~nm}$, $15 \mathrm{~nm}$ or $20 \mathrm{~nm}$, in order to study the influence of this parameter on the simulation results.

For the boundary conditions which must be applied to the simulation, two layers of atoms at the bottom of the work material and the four layers of atoms in the left side of the workpiece are kept fixed. Also, the initial temperature of the workpiece is $293 \mathrm{~K}$ and the simulation time step is $10 \mathrm{fs}$. The simulation time step is chosen to be very small in order to reduce the calculation error.
Three different atomic interactions in the Molecular Dynamics simulation of nanometric cutting processes need to be calculated, which are the interactions in the workpiece, the interactions in the tool and the interactions between the workpiece and the tool. In this study, the tool is considered to be rigid; diamond is harder than copper and so it can be assumed that the tool cannot be deformed and the atoms of the tool maintain the tool shape. In order to calculate the forces between the atoms of the workpiece, the interatomic Lennard-Jones potential is employed, which is a relatively simple potential. Morse potential is used in order to compare the use of the two different potentials and study their different results on the cutting forces. For the computation of the interactions between the workpiece and the tool, the Morse potential is used, as the constants of the potential for a system consisting of copper and diamond are known. For the interactions between the atoms of the workpiece, the constants are derived by Girifalco and Weizer (1959). Pei et al. (2006) suggested that the equilibrium distance between atoms shouls take the value of $r_{o}=2.78 \AA$, which they found that must be used in order to make the equilibrium lattice constant and equal to $3.62 \AA$. For the interactions between the workpiece and the tool, the constants are those proposed by Zhang and Tanaka (1997). All Morse potential constants are shown in Table 1.

In order to have an appropriate simulation and compute the wanted quantities, a Matlab ${ }^{\circledR}$ code is developed, in which three steps are followed, in general. The first step is the initialization phase, called pre-processing. The second step is the most basic step and this is the solver of the equations. The last step is the production of the output of the run or the post-processor.

At the first step, the characteristics of the system are initialized, which are those of the workpiece and the cutting tool. Both for the workpiece and cutting tool, the number of atoms is defined, the wanted geometry and the initial positions at the beginning of the simulation. The initial positions are depended on the crystal structure of the material used. Additionally, the initial velocities of the atoms are specified, which depend on the initial temperature of the system and are chosen in such a way so that the total momentum of the system is zero; the initial accelerations of the atoms are zero. 


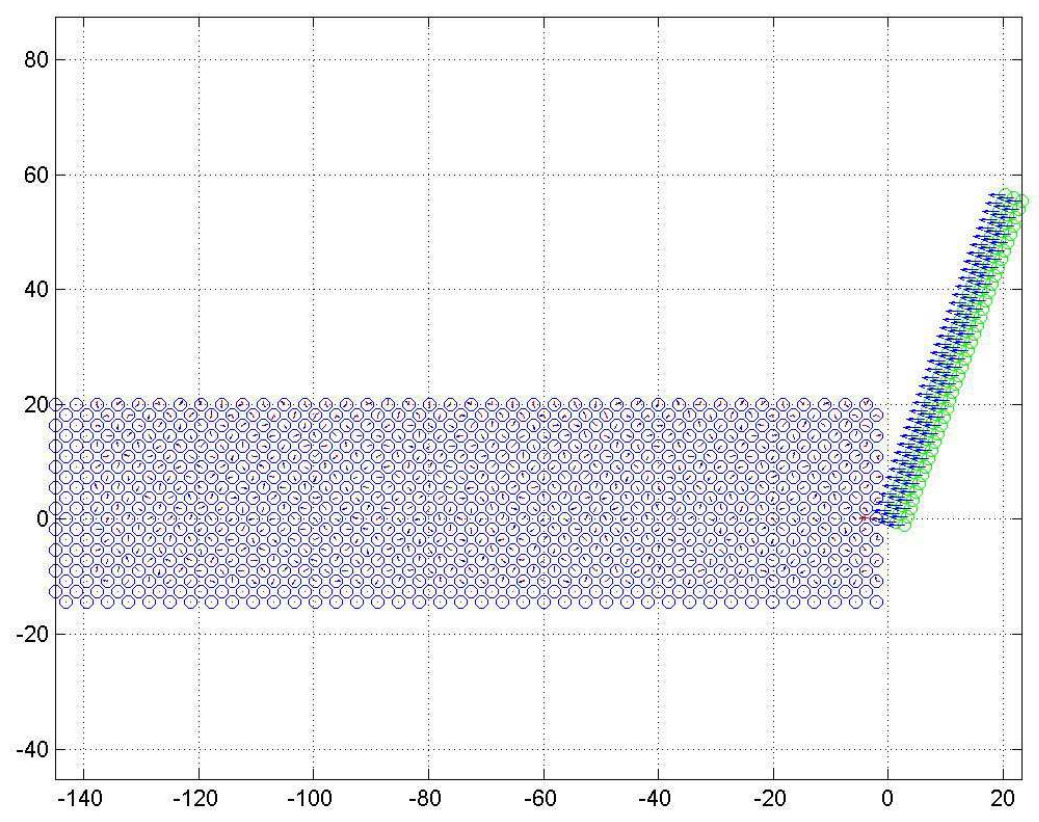

Fig. 1. Molecular dynamics' simulation model set-up

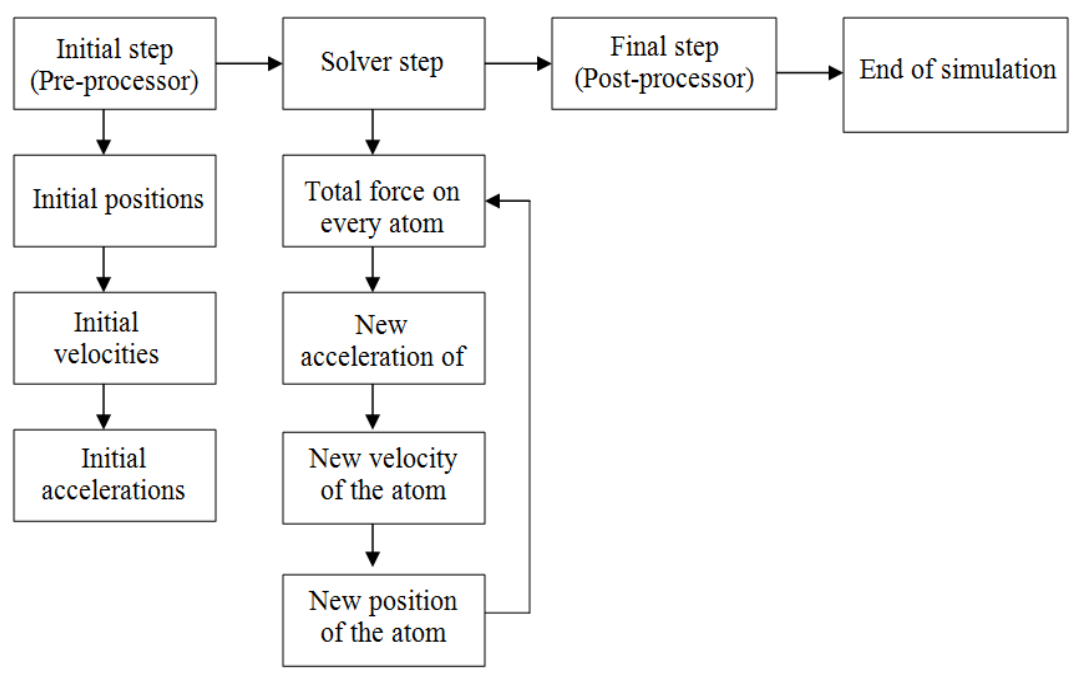

Fig. 2. Flow diagram of the simulation method

Table 1. Value of constants for the Morse potential for $\mathrm{Cu}-\mathrm{Cu}$ and $\mathrm{Cu}-\mathrm{C}$ interactions

\begin{tabular}{lll}
\hline QUANTITY & $\mathrm{Cu}-\mathrm{Cu}$ & $\mathrm{Cu}-\mathrm{C}$ \\
\hline $\mathrm{D}(\mathrm{eV})$ & 0.3429 & 0.0870 \\
$\alpha\left(\AA^{-1}\right)$ & 1.3588 & 5.1400 \\
$\mathrm{r}_{\mathrm{e}}(\AA)$ & 2.8660 & 2.0500 \\
\hline
\end{tabular}

At the second step, the equations of motion are integrated and solved at a repeating procedure. In every repeat, the following logic is adopted: The interaction force on every atom of the system is computed. For each atom, this force is the sum of all the forces that are being exerted on the atom from its neighbors in a specific distance $r_{c}$. After the total force that is being exerted on a specific atom for a specific moment is computed, the acceleration of the atom is calculated using the equations of motion. After this, the integration of motion equations follows, using a 
leapfrog-type numerical method, from which the velocities and the positions of each atom for every step is derived. This way, new forces on each atom and all the wanted quantities are calculated, using the measurements of the last step of the simulation. In every step of integration, the simulation continuues and the cutting tool removes material from the workpiece. This procedure ends when the wanted material has been removed from the workpiece. At every repeat, all measurements are saved in order to be collected at the end of the simulation. The above procedure is shown in the following diagram of Fig. 2.

\section{RESULTS AND DISCUSSION}

In the initial studies, Lennard-Jones potential is used in order to compute the forces between the atoms of the copper workpiece. Initially, the influence of the depth of cut on the chip formation and the smoothness of the machined surface are studied. Various values for the depth of cut, namely $10 \AA, 15 \AA$ and $20 \AA$, are tested. The respective simulations are shown in Fig. 3 (a) to (c) for a tool with tool rake angle of $0^{\circ}$. From the simulation results, it can be concluded that the material of the workpiece is deformed as the tool moves on it in a similar way to conventional machining processes. The chip follows the shape of the tool depending on the tool rake angle, the unwanted material is removed and the machined surface takes the wanted form. Phenomena like elastic recovery of the material are obvious; atoms of uncut
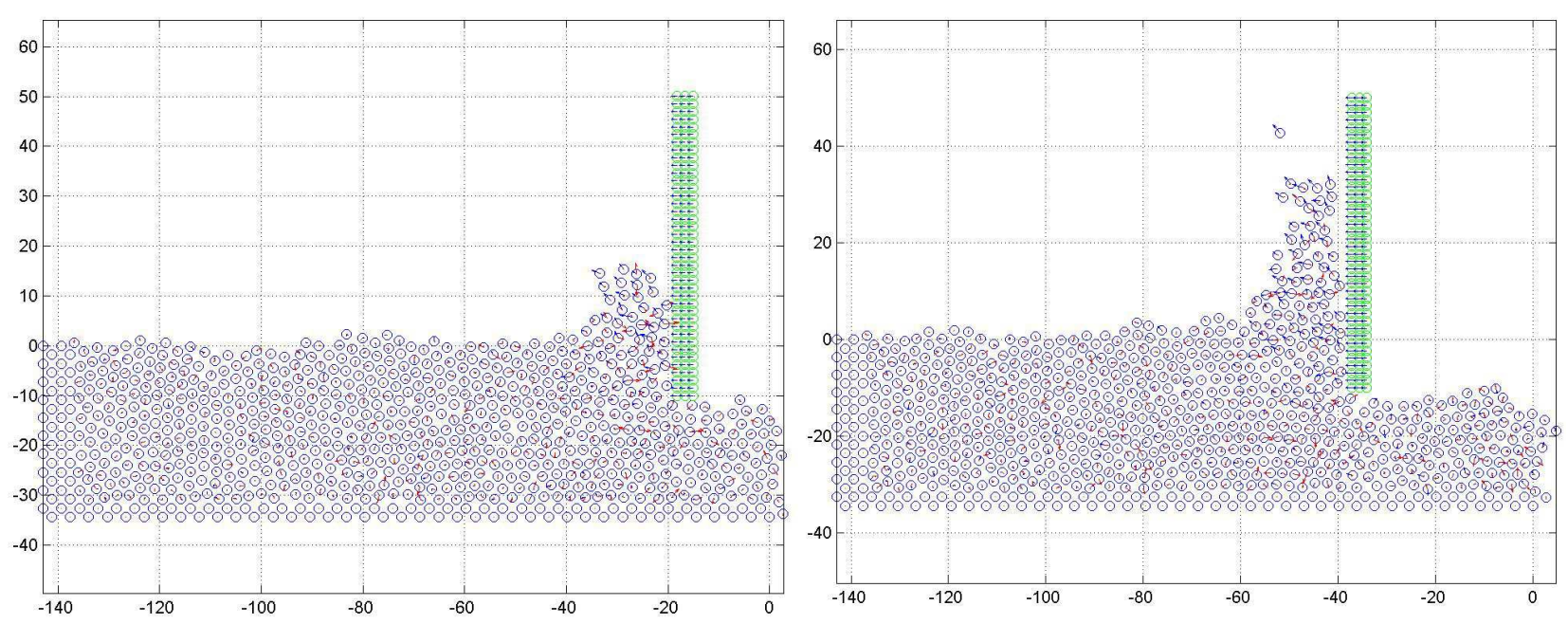

(a)

material that are far away from the tool are not affected by the machining process.

For different values of depth of cut, the simulation results are similar, but with some distinct differences; for higher values of depth of cut, machined surface becomes rougher. Furthermore, many dislocations and voids appear in the cutting region as the depth of cut becomes larger.

In the presented Molecular Dynamics simulations, cutting forces during the cutting process can be predicted. Cutting forces are the sum of the interatomic forces between the atoms of the workpiece and the atoms of the tool; tangential force $F_{x}$ and normal force $F_{y}$ can be calculated. In Fig. 4, the influence of the depth of cut on both $F_{x}$ and $F_{y}$ forces of the cutting process for rake angle of $0^{\circ}$ is depicted. It is worth noticing that the tangential cutting force $F_{x}$ is always higher than the normal cutting force $F_{y}$. Furthermore, when depth of cut increases, both forces increase, too. The variation of the ratio of the normal force to the tangential force with the depth of cut is presented in Fig. 5, where this ratio is increasing with the increasing of depth of cut.

Next, different values for the tool rake angle are used, namely $10^{\circ}, 20^{\circ}$ and $30^{\circ}$. The respective simulations are shown in Fig. 6a-c with depth of cut equal to $10 \AA$. Once again, from simulation results, cutting process is similar to conventional machining processes and chip takes different shape as the tool rake angle changes. As tool rake angle increases, fewer dislocations and voids appear in the cutting region. 

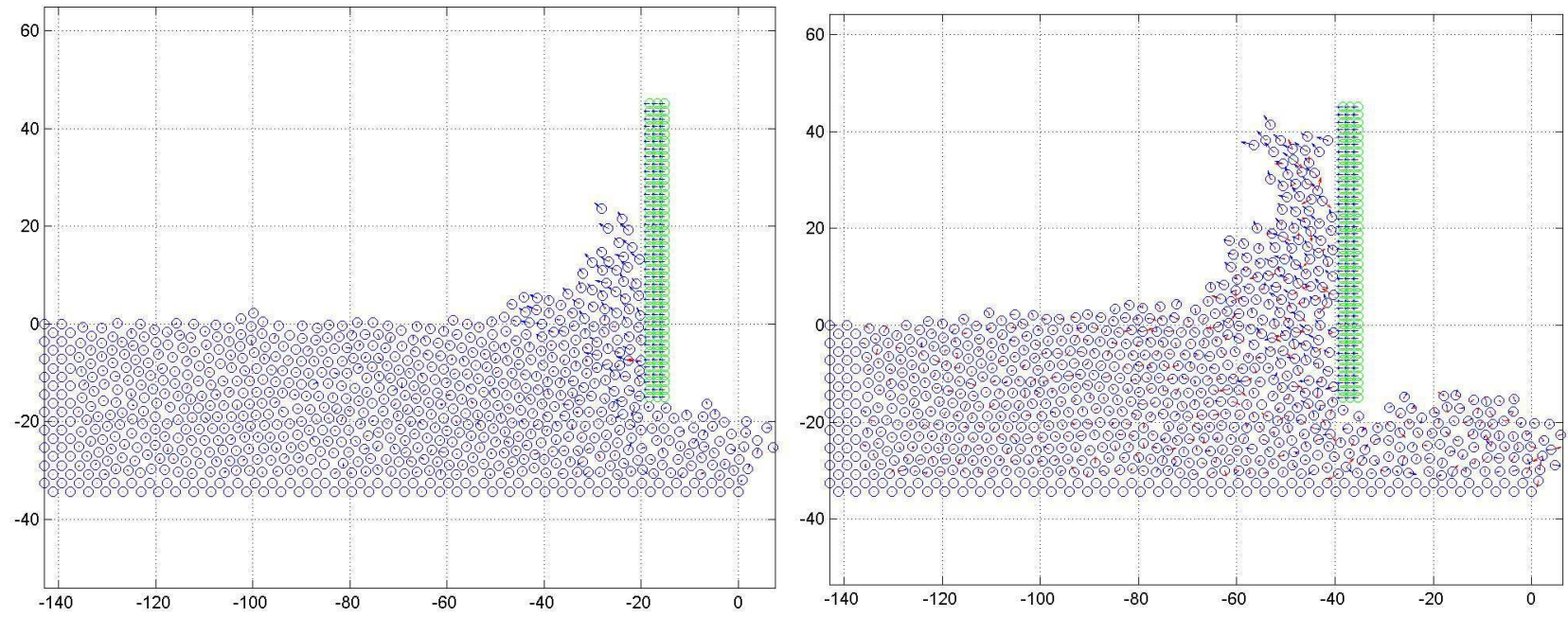

(b)
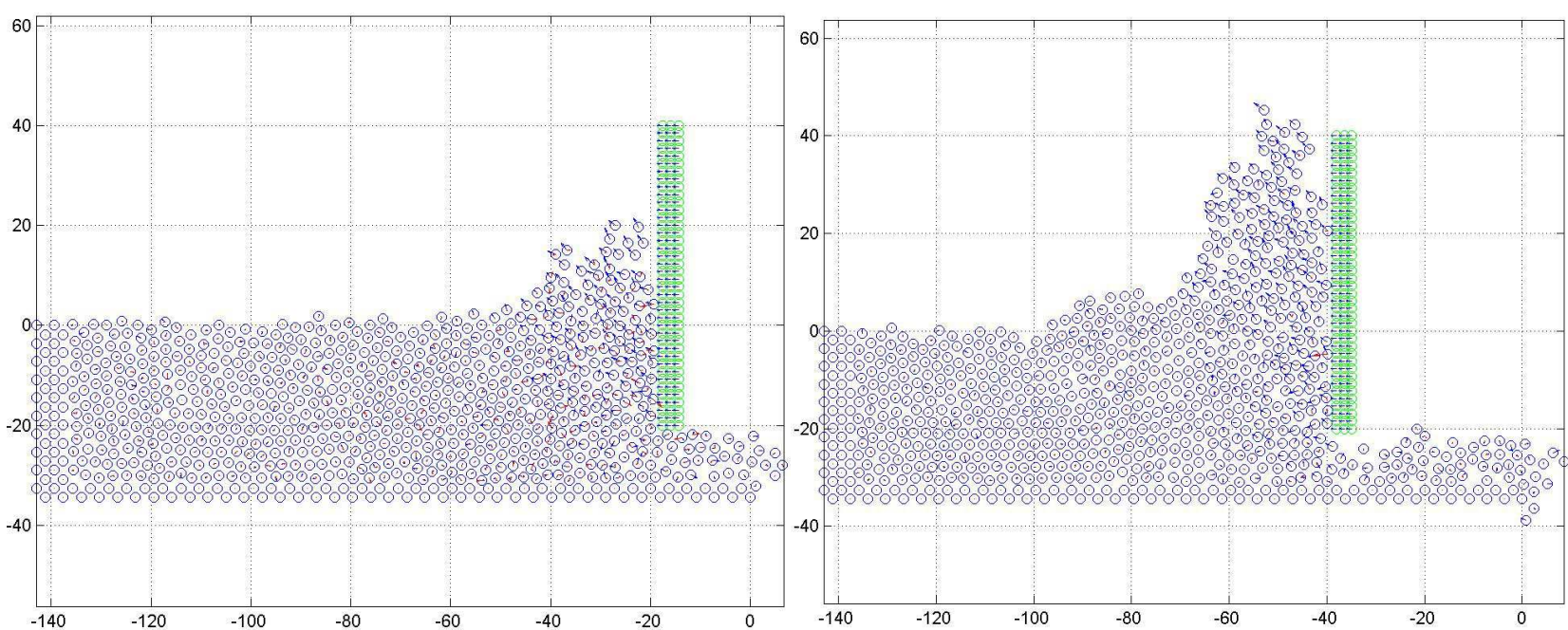

(c)

Fig. 3. Simulation results for a tool rake angle of 0 o and with depth of cut (a) $10 \AA$, (b) $15 \AA$ and (c) $20 \AA$

In Fig. 7, the influence of tool rake angle on both $\mathrm{F}_{\mathrm{x}}$ and $\mathrm{F}_{\mathrm{y}}$ forces is depicted for depth of cut equal to $10 \AA$. Tangential cutting force $F_{x}$ is always higher than normal cutting force $F_{y}$, as anticipated. The variation of the ratio of the normal force to the tangential force with tool rake angle can be seen in Fig. 8; the ratio is decreasing with the increase in the depth of cut.

In the previous simulations Lennard-Jones potential was used. However, the difference on the simulation results using either the Lennard-Jones potential or the Morse potential can be investigated. Respective simulations with the use of Morse potential are shown in Fig. 9 with various tool rake angles and a specific depth of cut at $10 \AA$.

From the simulation results, it can be seen that there no significant difference on the chip morphology and the smoothness of the machined surface for the same cutting conditions using either the Lennard-Jones or Morse potential. However, one difference is that with the Morse potential, the machined surface appears to be smoother than by using the Lennard-Jones potential; also, the dislocations and the voids are fewer. In Fig. 10a and b, various cutting forces appearing with the use of the two different potentials are presented. 
Angelos P. Markopoulos et al. / American Journal of Nanotechnology 5 (2): 17-26, 2014

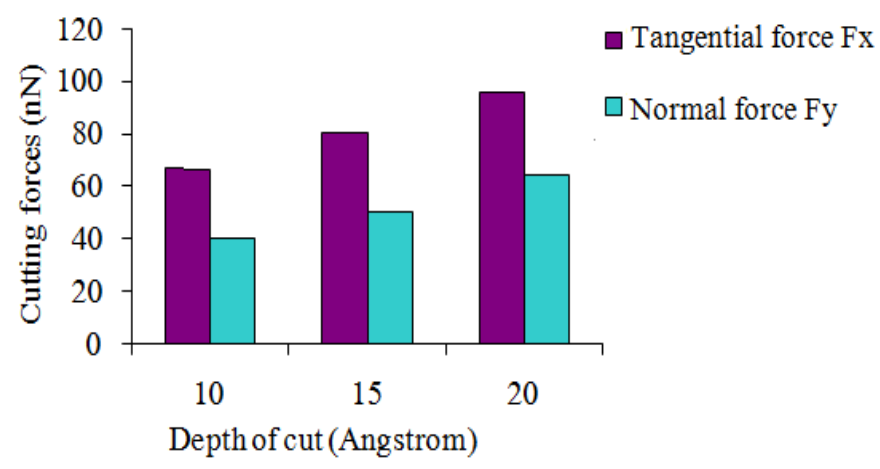

Fig. 4. Variation in the cutting forces for three different depths of cut, for tool rake angle $0^{\circ}$

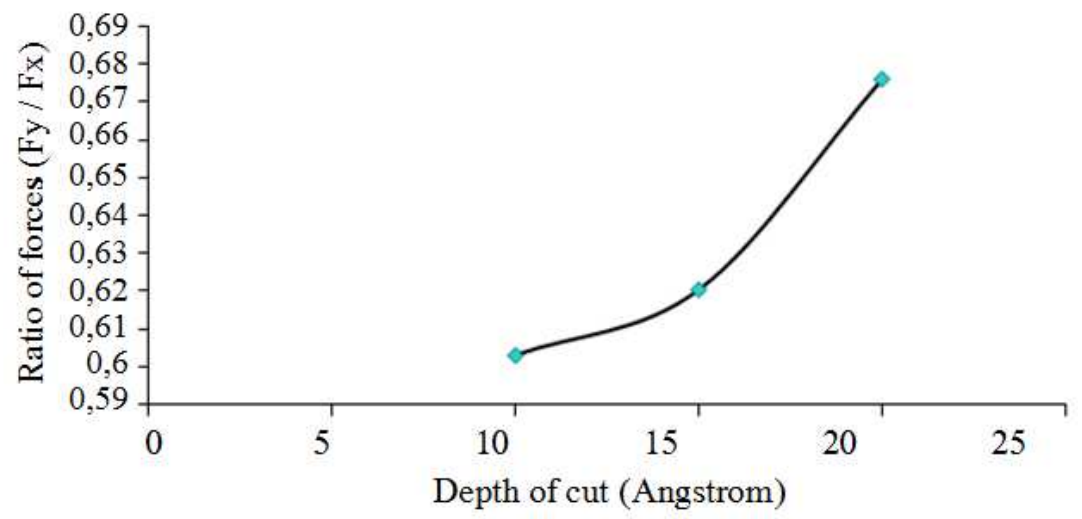

Fig. 5. Variation in the ratio of normal to tangential cutting force for different depths of cut and tool rake angle 0
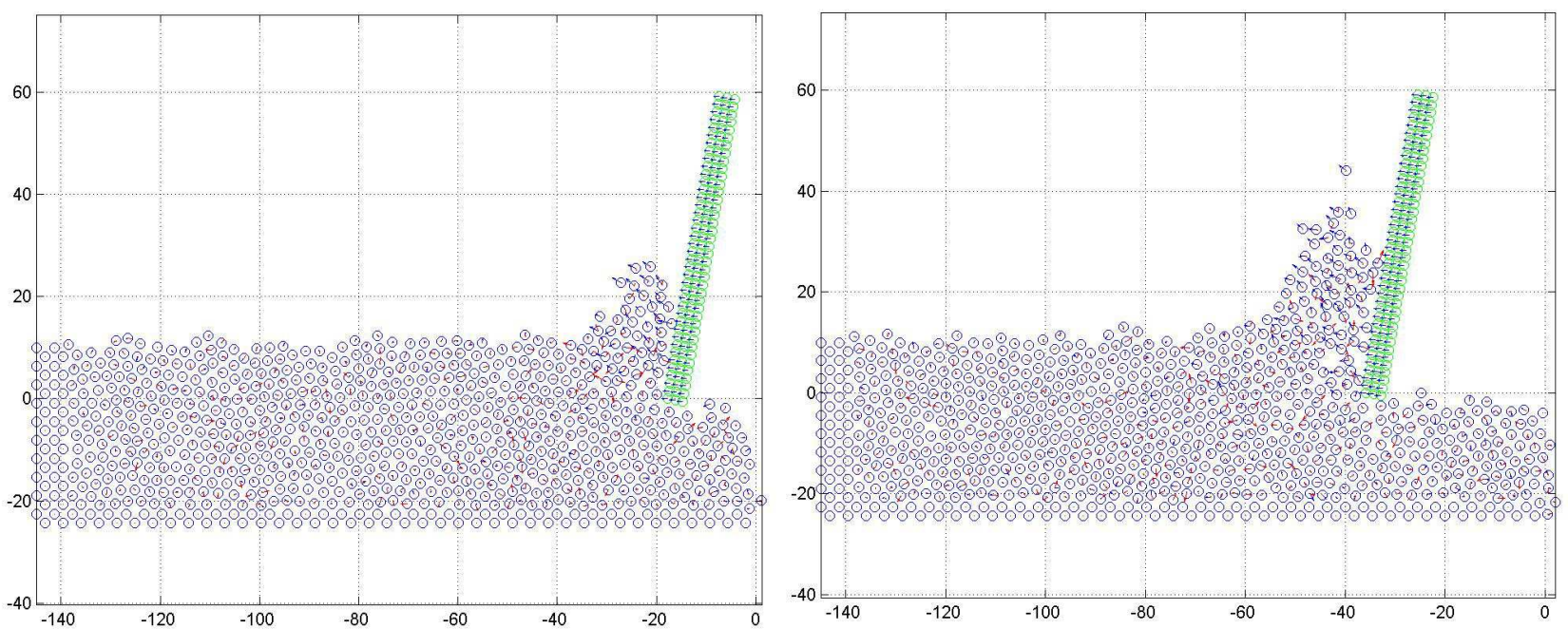

(a) 
Angelos P. Markopoulos et al. / American Journal of Nanotechnology 5 (2): 17-26, 2014
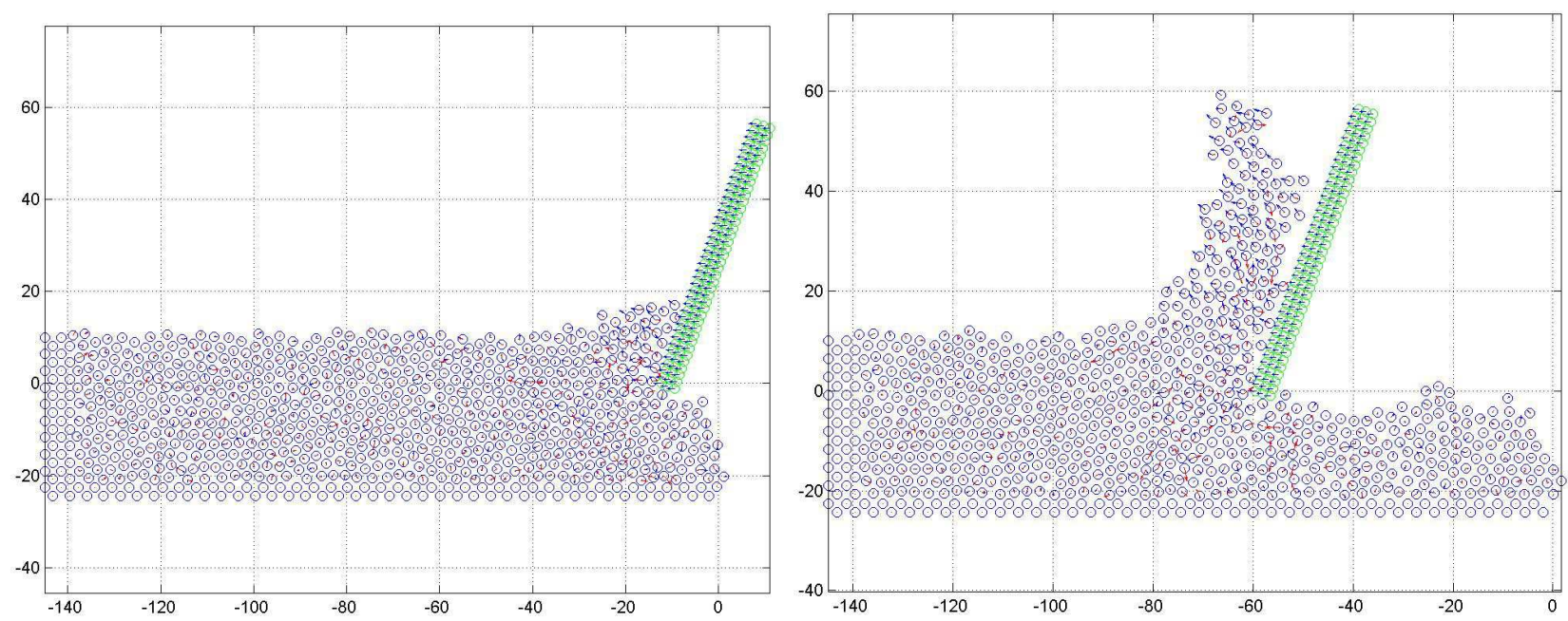

(b)
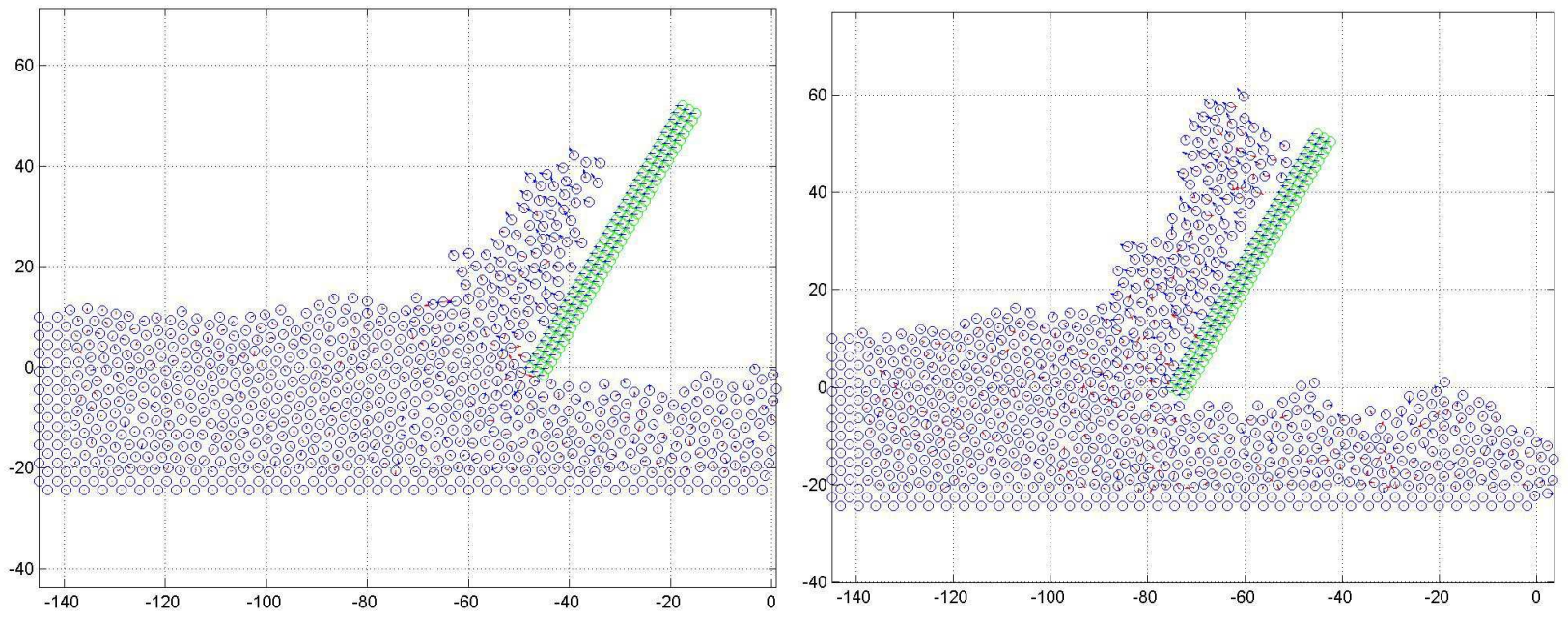

(c)

Fig. 6. Simulation results with for a depth of cut of $10 \AA$ and tool rake angle (a) $10^{\circ}$ (b) $20^{\circ}$ and (c) $30^{\circ}$

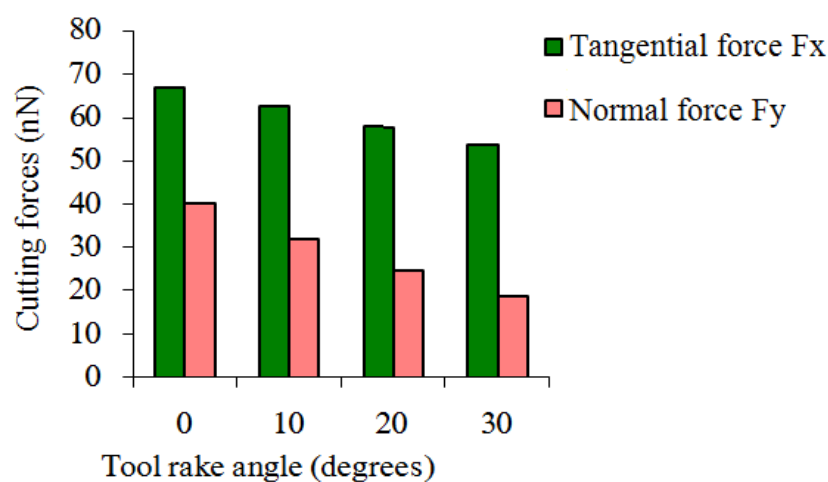

Fig. 7. Variation of the cutting forces for different tool rake angles for depth of cut equal to $10 \AA$ 


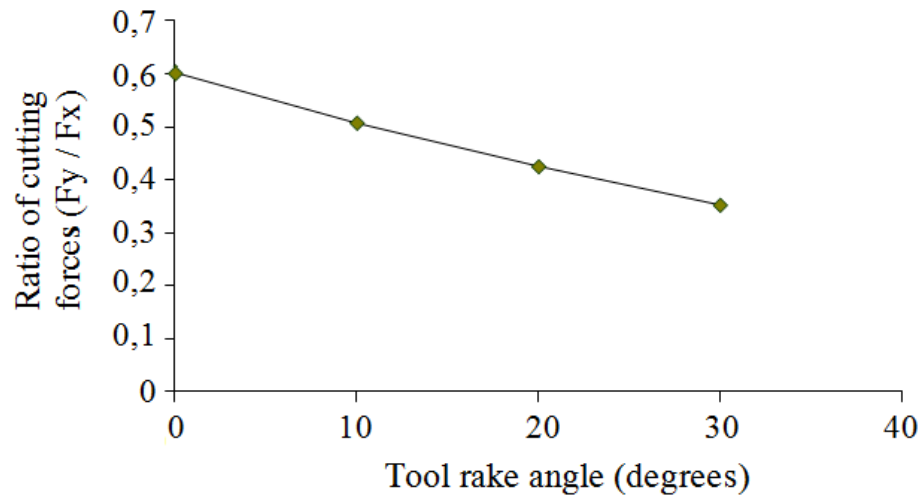

Fig. 8. Variation of the ratio of the normal to the tangential cutting force for various tool rake angles for a depth of cut of $10 \AA$

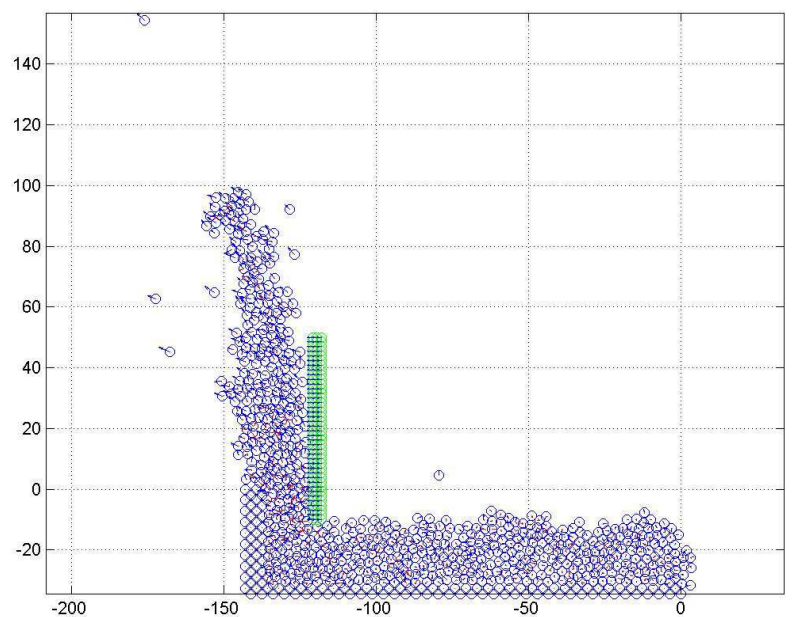

(a)

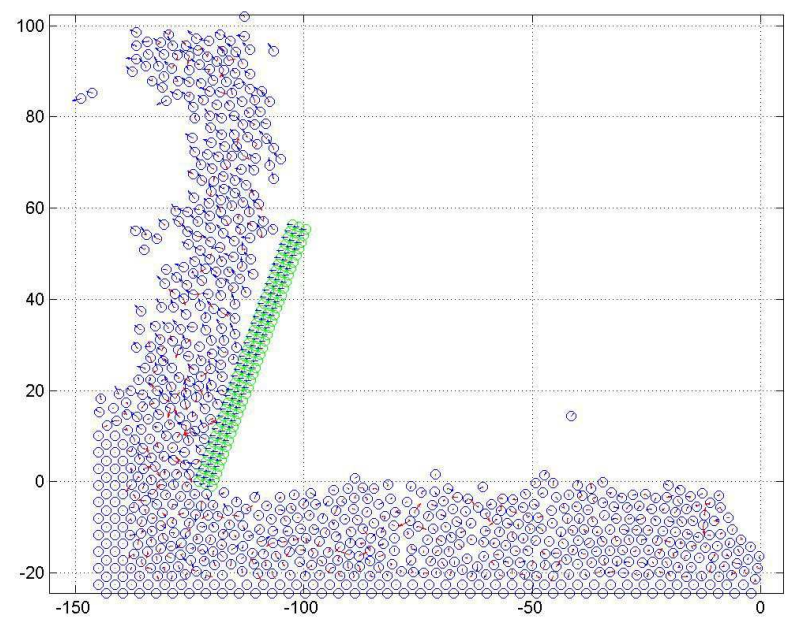

(c)

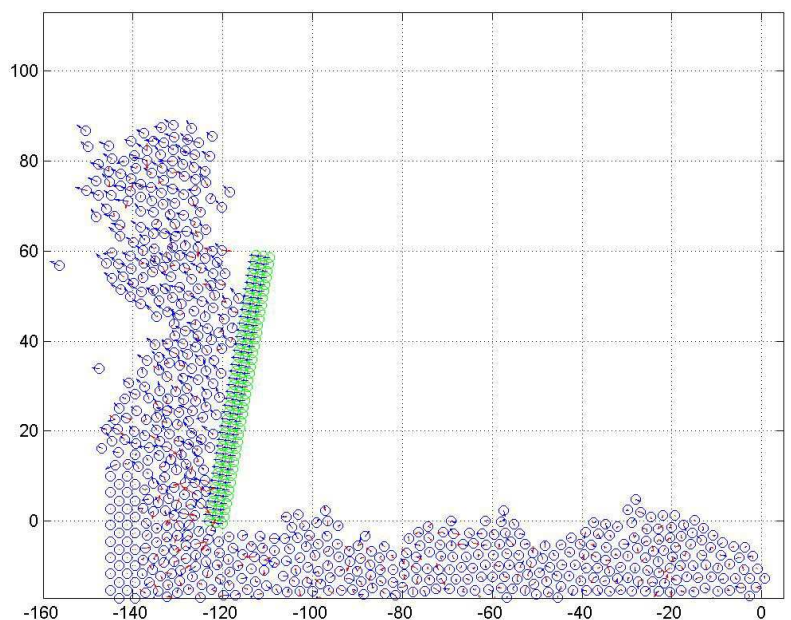

(b)

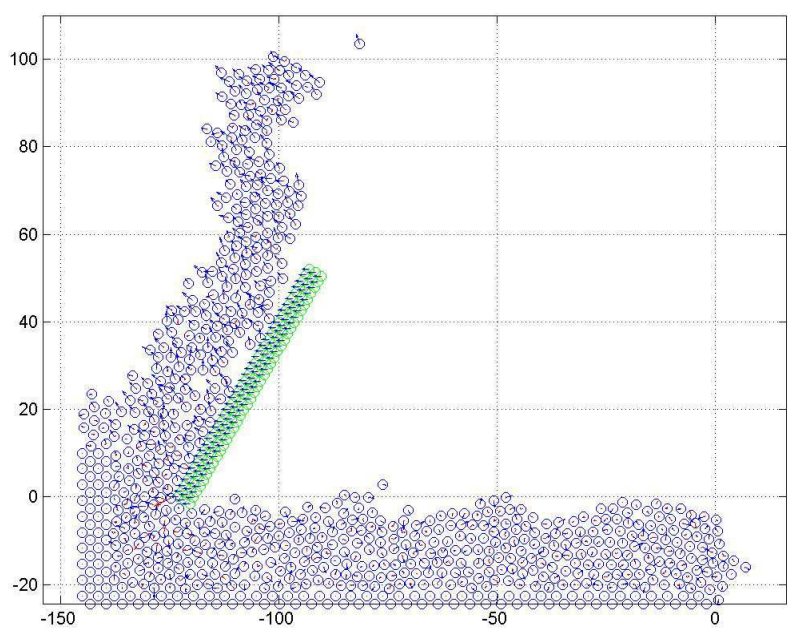

(d)

Fig. 9. Simulation results using Morse potential for depth of cut of $10 \AA$ and (a) $0^{\circ}$ (b) $10^{\circ}$ (c) $20^{\circ}$ and (d) $30^{\circ}$ tool rake angle 


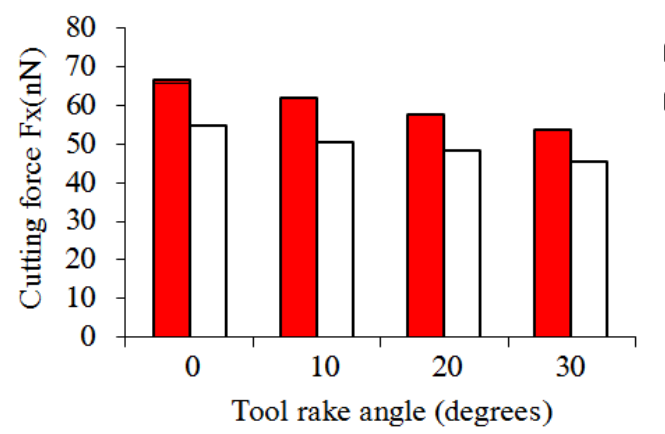

口 Lennard-Jones

$\square$ Morse

(a)

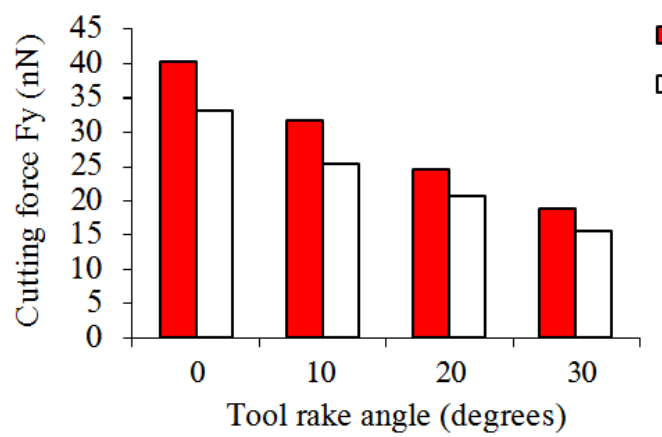

Lennard-Jones

$\square$ Morse

(b)

Fig. 10. Variation of (a) tangential and (b) normal cutting force for various tool rake angles, for depth of cut $10 \AA$ for Lennard-Jones and Morse potentials

It can be seen that under Morse potential both tangential and normal cutting forces are smaller compared to the ones calculated with Lennard-Jones potential. This happens due to the different nature of the two potentials and the different way the metallic bond is being computated. This is an important observation when using Molecular Dynamics simulation to study the nanometric cutting process of copper with a diamond tool.

\section{CONCLUSION}

Simulations of nanometric cutting process of copper with diamond tool for different cutting conditions, using Molecular Dynamics method were carried out. The following results were obtained:

- Depth of cut has an influence on chip formation and roughness of the machined surface. By increasing the depth of cut, the machined surface becomes rougher and many dislocations and voids appear in the cutting region

- Cutting forces are influenced by the different depths of cut. By increasing the depth of cut from $10 \AA$ to
$20 \AA$, cutting forces $\mathrm{F}_{\mathrm{x}}$ and $\mathrm{F}_{\mathrm{y}}$ also increase considerably. The same happens with the ratio of normal to tangential cutting force

- Tool rake angle has also an influence on chip morphology and roughness of the machined surface. By increasing tool rake angle from $0^{\circ}$ to $30^{\circ}$, the machined surface becomes smoother and the atoms in the cutting region are still close to one another

- Cutting forces appear to be influenced by the variation of the tool rake angle, as well. As tool rake angle increases, cutting forces decrease considerably and the same happens with the ratio of normal to tangential cutting force

- There are no significant differences in chip formation and roughness of the machined surface between the Lennard-Jones and Morse potential simulations. However, with Morse potential, the machined surface appears to be smoother

- There is a significant influence on the cutting forces between the two different potentials. Morse potential results in lower cutting forces than Lennard-Jones potential for the same cutting conditions 


\section{ADDITIONAL INFORMATION}

\subsection{Funding Information}

The authors have no support or funding to report.

\subsection{Author's Contributions}

All authors equally contributed in this work.

\subsection{Ethics}

This article is original and contains unpublished material. The corresponding author confirms that all of the other authors have read and approved the manuscript and no ethical issues involved.

\section{REFERENCES}

Girifalco, L.A. and V.G. Weizer, 1959. Application of the Morse potential function to cubic materials. Phys. Rev., 114: 687-690. DOI: 10.1103/PhysRev.114.687

Ikawa, N., S. Shimada, H. Tanaka and G. Ohmori, 1991. An atomistic analysis of nanometric chip removal as affected by tool-work interaction in diamond turning. Ann. CIRP, Manuf. Technol., 40: 551-554. DOI: $10.1016 / \mathrm{S} 0007-8506(07) 62051-4$

Kim, J.D. and C.H. Moon, 1996. A study on the cutting mechanism of microcutting using molecular dynamics. Int. J. Adv. Manuf. Technol., 11: 319-324. DOI: $10.1007 / \mathrm{BF} 01845690$

Komanduri, R., N. Chandrasekaran and L.M. Raff, 1998. Effect of tool geometry in nanometric cutting: A molecular dynamics simulation approach. Wear, 219: 84-97. DOI: 10.1016/S0043-1648(98)00229-4

Komanduri, R., N. Chandrasekaran and L.M. Raff, 1999. Some aspects of machining with negative-rake tools simulating grinding: A molecular dynamics simulation approach. Phil. Mag. Part B., 79: 955-968. DOI: $10.1080 / 13642819908214852$
Komanduri, R., N. Chandrasekaran and L.M. Raff, 2000. MD simulation of nanometric cutting of single crystal aluminum-effect of crystal orientation and direction of cutting. Wear, 242: 60-88. DOI: $10.1016 / \mathrm{S} 0043-1648(00) 00389-6$

Komanduri, R., N. Chandrasekaran and L.M. Raff, 2001. Molecular Dynamics (MD) simulation of uniaxial tension of some single-crystal cubic metals at nanolevel. Int. J. Mech. Sci., 43: 2237-2260. DOI: 10.1016/S0020-7403(01)00043-1

Komanduri, R. and L.M. Raff, 2001. A review on the molecular dynamics simulation of machining at the atomic scale. Proc. Instn Mech. Eng, Part B, J. Eng. Manuf., 215: 1639-1672.

DOI: $10.1177 / 095440540121501201$

Rapaport, D.C., 2004. The Art of Molecular Dynamics Simulation. 1st Edn., Cambridge University Press, New York, ISBN-10: 0521825687, pp: 549.

Pei, Q.X., C. Lu, F.Z. Fang and H. Wu, 2006. Nanometric cutting of copper: A molecular dynamics study. Comput. Mater. Sci., 37: 434-441. DOI: 10.1016/j.commatsci.2005.10.006

Shimada, S., 1995. Molecular dynamics analysis of nanometric cutting process. Int. J. Japan, Soc. Prec. Eng, 29: 283-286.

Ye, Y.Y., R. Biswas, J.R. Morris, A. Bastawros and A. Chandra, 2003. Molecular dynamics simulation of nanoscale machining of copper. Nanotechnology, 14: 390-396. DOI: 10.1088/0957-4484/14/3/307

Zhang, L. and H. Tanaka 1997. Towards a deeper understanding of wear and friction on the atomic scalea molecular dynamics analysis. Wear, 211: 44-53. DOI: 10.1016/S0043-1648(97)00073-2 